\title{
Análisis filosófico sobre el currículum de Educación Física en Chile Philosophical analysis of the Physical Education curriculum in Chile
}

\author{
Felipe Nicolás Mujica Johnson \\ Universidad Autónoma de Chile (Chile)
}

\begin{abstract}
Resumen. La Educación Física moderna surge con la fundamentación de la filosofía moderna, en la cual destaca la corriente racionalista y positivista. De este modo, las primeras bases curriculares se desarrollaron con el enfoque conductista de la pedagogía por objetivos, orientado por la estandarización, la univocidad ideológica, la mirada objetivista de la corporalidad, la reproducción de contenidos didácticos, el aprendizaje pasivo, la rigidez, el discurso de neutralidad y la obsesión por la eficacia. Después de varias décadas de aquella perspectiva surgieron en el mundo y, sobre todo, en Europa, perspectivas teóricas que propusieron bases filosóficas posmodernas. Orientadas por la relatividad, la pluralidad ideológica, la mirada subjetiva de la corporalidad, la flexibilidad, la contextualización, el posicionamiento histórico-cultural y el aprendizaje activo. Aquella mirada posmoderna no estaría muy reflejada en el contexto escolar chileno, de modo que este ensayo se propuso analizar desde la perspectiva filosófica algunos aspectos problemáticos que existirían en torno al currículum de Educación Física escolar en Chile. Los resultados del análisis muestran tres aspectos curriculares conflictivos, asociados al SIMCE de Educación Física, al enfoque orientado a la salud que propone el nuevo nombre de la asignatura y al diferente paradigma curricular entre la formación inicial docente con la realidad escolar. Este conocimiento refleja que existen importantes desafíos curriculares en torno a la asignatura y el contexto estudiado, sobre todo, para integrar los nuevos saberes contemporáneos. Aquello también exige un amplio debate en torno a la materia y una amplia participación del profesorado como del alumnado.
\end{abstract}

Palabras clave: Filosofía, modernidad, posmodernidad, currículum, educación física.

\begin{abstract}
Modern Physical Education arises with the foundation of modern philosophy, in which the rationalist and positivist current stands out. Thus, the first curricular bases were developed with the behaviorist approach of pedagogy by objectives, oriented by standardization, ideological univocity, the objectivist view of corporeality, the reproduction of didactic contents, passive learning, rigidity, the discourse of neutrality and the obsession with efficiency. After several decades of that perspective, theoretical perspectives emerged in the world, especially in Europe, that proposed postmodern philosophical bases. Oriented by relativity, ideological plurality, the subjective view of corporeality, flexibility, contextualization, historical-cultural positioning and active learning. That postmodern look would not be very much reflected in the Chilean school context, so this essay was proposed to analyze from a philosophical perspective some problematic aspects that would exist around the school Physical Education curriculum in Chile. The results of the analysis show three conflicting curricular aspects, associated with the SIMCE of Physical Education, the health-oriented approach proposed by the new name of the subject and the different curricular paradigm between initial teacher training and school reality. This knowledge reflects that there are important curricular challenges around the subject and the context studied, above all, to integrate new contemporary knowledge. This also requires a broad debate on the subject and a wide participation of both teachers and students.
\end{abstract}

Key words: Philosophy, modernity, postmodernity, curriculum, physical education.

\section{Introducción}

En este ensayo se presentará, el vínculo que existe entre la filosofía y el currículum educativo, lo que en términos epistemológicos sería parte de la Filosofía de la Educación (Dewey, 2004; García, 2014).Y, en términos más específicos, de la Filosofía de la Educación Física (EF) (García, 1999; Mujica, 2020a, 2020b; 2021a; Mujica \& Orellana, 2021; Stolz, 2013, 2014), pues, se abordará principalmente esta última disciplina pedagó-

Fecha recepción: 19-08-21. Fecha de aceptación: 01-12-21

Felipe Nicolás Mujica Johnson

felipe.mujica@uautonoma.cl gica. Sobre todo, desde las problemáticas que existirían en la actualidad en el currículum escolar chileno (Matus, 2016; Moreno, 2018; Mujica \& Inostroza, 2020; Oliva, 2008). De acuerdo con los planteamientos teóricos de Gimeno-Sacristán (2010), el currículum surge con la finalidad de controlar el proceso educativo con finalidades filosóficas y políticas concretas. Por ello, aunque no siempre se haya visibilizado, el currículum educativo no es neutral. Con relación a esta última característica, Matus (2016) explica que hay discursos pedagógicos que intentan aparentar una neutralidad curricular para simplificar y reducir las posibilidades de transformación social. Aquella mirada, como se verá más adelante, es parte de una de las corrientes filosóficas de la teoría 
curricular. Con base en lo planteado y para otorgar mayor precisión conceptual, en este trabajo se entiende el currículum como un proyecto o plan pedagógico que regula el proceso escolar de enseñanza-aprendizaje (Gimeno-Sacristán, 2010).

El currículum escolar, como proyecto regulador, presenta múltiples directrices que orientan la actividad pedagógica, las que, para ser entendidas, en un amplio sentido del término comprensión, requieren de un previo entendimiento de las bases filosóficas que las sostienen o fundamentan. De lo contrario, se generaría un entendimiento superficial del tema curricular, lo cual puede favorecer una perspectiva reproductiva y carente de pensamiento crítico frente a la materia (CasillasGutiérrez, 2019). En relación con lo planteado, diferentes estudios curriculares en EF han reconocido la presencia de dichas características, lo que se traduce en una EF de corte tecnocrático o técnico (Kirk, 2010, 2017; Lawson, 2009, 2018, 2019; Moreno, Gamboa \& Poblete, 2014; Moreno \& Poblete, 2015). Cabe mencionar que un modelo curricular tecnocrático, tecnológico-positivista o conductista se caracteriza por una mirada cerrada y rígida del proceso enseñanza-aprendizaje (Mujica \& Concha, 2015; Torres, 2002). Asimismo, se ha planteado que es univocista-objetivista (Monzón, 2010), pues, solamente acepta una sola interpretación de los sucesos pedagógicos. En términos más específicos, en dicho modelo tecnocrático el currículum escolar «se define como una planificación desde una base conductual que busca la obtención de productos observables, medibles y cuantificables. Se entiende también como la organización de medios y fines orientados a la consecución de objetivos observables, medibles y cuantificables» (Monzón, 2010, p. 50). Por esto mismo, aquel modelo tecnocrático se identifica con la pedagogía por objetivos, la cual encuentra sus raíces, en buena parte, en el pensamiento de Hilda Taba y Ralph Tyler, dos docentes estadounidenses que vieron la necesidad de sistematizar la enseñanza en las escuelas por medio de conductas observables (Esparza y Zabala, 2015). Aquella pedagogía por objetivos significó un avance pedagógico para mediados del siglo XX, pero, como bien explica GimenoSacristán (2002), se le otorgó una excesiva importancia a la eficiencia y se asumió una mirada cientificista de la educación. Para comprender bien aquel modelo es necesario entender sus fundamentos teóricos que tienen implícitos, ya que no es un modelo ingenuo, sino que representa una interpretación científica del mundo. Por lo mismo, es fundamental someterla a diferentes análisis críticos que consideren sus bases teóricas.
Anteriormente se señaló que el modelo curricular tecnocrático se encuentra asociado al positivismo, de modo que es necesario revisar algunos de los postulados de aquella concepción filosófica y que se refleja en las ciencias particulares, como la ciencia de la educación. De acuerdo con González y Hernández (2014), el positivismo es una de las corrientes filosóficas que ha influenciado las ciencias de la educación en los últimos 150 años. La filosofía positivista, según explican Iskandar \& Rute (2002), se inicia formalmente en el siglo XIX con las ideas del francés Augusto Comte, no obstante, agregan que sus raíces se pueden hallar desde antes de aquel autor. Asimismo, señalan que surge influenciado por el pensamiento de la filosofía moderna y racionalista, con énfasis, del pensamiento kantiano. En la época moderna hubo variadas personalidades de la filosofía que influenciaron el pensamiento de aquel tiempo, pero algunos destacados representantes son René Descartes (2003), Immanuel Kant (1989), Jean-Jacques Rousseau (2011) y John Locke (1984). En aquel periodo moderno hubo dos grandes corrientes filosóficas que se enfrentaron por el poder intelectual de la época, el racionalismo y el empirismo (Cabedo, 2006). El racionalismo sostiene que la razón o la mente humana es la única fuente de la verdad, mientras que el empirismo señala que la fuente de la verdad es la experiencia o los hechos observables (Cabedo, 2006; Ferrater, 1994; Rojas, 2006). No obstante, cabe destacar, que el empirismo también le otorga mucha importancia a la razón humana y a su superioridad frente a otros ámbitos del ser humano (Ferrater, 1994; Locke, 1984). En cuanto a los aportes intelectuales de cada corriente, el racionalismo se encargó de desarrollar, en buena parte, conocimientos metafísicos, mientras que el empirismo conocimiento de ciencias particulares o lo que comúnmente se reconoce como conocimiento científico (Cabedo, 2006; Sánchez, 2010; Tredici, 1958). Es importante destacar que la filosofía también se reconoce como ciencia, pero una ciencia general (Mujica, 2021a; Stein, 2005; Von Hildebrand, 2000), de modo que el conocimiento del racionalismo también podría ser denominado conocimiento científico. El positivismo como corriente filosófica surge del empirismo y el racionalismo, donde sus fundamentos de las ciencias particulares, cargados de un fuerte acento cuantitativo y muy presentes en las ciencias naturales, se intentaron generalizar a la comprensión general del mundo (Iskandar \& Rute, 2002; Pérez, 2015; Tredici, 1958).

El currículum de EF también se asocia a otra de las corrientes filosóficas modernas, que es el idealismo o 
racionalismo. Esto se aprecia, principalmente, en el desarrollo de una concepción dualista que fragmenta al ser humano en cuerpo y alma, como dos aspectos no se encuentran integrados. Esta concepción fue heredada de la ontología platónica-cartesiana (Contreras, 1998; Gallo, 2009; Mujica, 2021b), donde el alma representaba la inteligencia y la razón, aspectos que no identificaban a la corporalidad. Dicha concepción idealista fomentó una mirada higienista o biomédica de la EF (Moreno, 2018; Pastor, 2005), donde la clase de aquella asignatura se enfocaba en el entrenamiento de la condición física y se desentendía de una educación integral, puesto que no se enfocaba en las emociones, la comprensión de la actividad físico-deportiva y otros aspectos motrices. Esto provocó que se desarrollaran nuevas metodologías de aprendizaje en EF, como la enseñanza comprensiva del deporte (Bunker \& Thorpe, 1982; Castejón, 2010; López-Ros, 2016). Cabe destacar, que existen diferentes idealismos filosóficos, de modo que no todos comparten el dualismo estricto de Platón (1988) y Descartes (2003). Una alternativa sería el dualismo aristotélicotomista, donde se presenta una mirada integrada entre cuerpo y alma (Beuchot, 2003; Mujica, 2021b).

Este ensayo plantea como tesis que la EF en Chile está en la actualidad fuertemente influenciada por las corrientes filosóficas tradicionales de la modernidad, es decir, por el positivismo y el racionalismo. Esta relación se fundamentará a partir de algunas problemáticas curriculares que serán expuesta de un modo más amplio. Por medio de estos planteamientos se espera poder ampliar las perspectivas en torno al currículum de EF y, con ello, poder fomentar nuevos estudios empíricos y teóricos sobre estos temas. En este sentido, los argumentos serán de corte teórico y, por supuesto, reflejan una mirada particular sobre el fenómeno curricular de la EF, la cual ha ser sometida a diferentes análisis críticos para contrastarla con otras perspectivas. También se hará énfasis en la teoría de la pedagogía por objetivos, puesto que tiene mucha relación con el actual currículum chileno (Mujica, 2020c). Sobre la influencia del pensamiento moderno racionalista y positivista en la teoría curricular, se ha caracterizado por «el predominio de la razón instrumental, la estandarización, la regulación de clases, razas, diferencias de género a través de formas rígidas de evaluación, clasificación y seguimiento» (Torres, 2002, p. 200). El positivismo es una filosofía que, en buena parte, es anti-filosófica, ya que rechaza las ideas concebidas de forma independiente de la experiencia. Aunque, siendo más precisos, es anti-metafísica, por lo que el filósofo ítalo-alemán Von
Hildebrand (2000) cuestiona que el positivismo sea llamado una filosofía propiamente tal. Dado que no aceptan postulados independientes de la realidad fáctica, como se ha mencionado anteriormente, promueve una supuesta neutralidad en su método, la cual también fue trasladada al currículum educativo (Matus, 2016). La pedagogía por objetivos también fue influenciada por factores históricos, como la revolución industrial y el utilitarismo que se vivió en buena parte de occidente, sobre todo, en Estados Unidos (Mujica \& Concha, 2015). De hecho, aquel lugar fue el origen donde se desarrolló dicho enfoque curricular. Con la pedagogía por objetivos la industria «se toma como esquema-patrón en la organización y desarrollo del currículo escolar» (Gimeno-Sacristán, 2002, p. 15). Asunto que, según especialistas de EF (Kirk, 2010; Lawson, 2018, 2019), ha sido perjudicial hasta la actualidad, puesto que reduce las posibilidades de aplicación de modelos pedagógicos innovadores, como el Modelo de Educación Deportiva (Molina et al., 2020; Segovia \& Gutiérrez, 2019; Siedentop, 1994).

La filosofía moderna siempre tuvo resistencia frente a sus postulados, sobre todo, frente al anhelo de ordenar estrictamente el mundo. Uno de los movimientos más reconocidos de aquella resistencia es el del romanticismo o idealismo alemán, que buscó reivindicar el sentido de libertad del ser humano frente al control del estado moderno (Arango, 2008; Vilanou, 2014). Asimismo, también hubo importantes referentes de la filosofía que combatieron la modernidad y han contribuido a la pérdida de su hegemonía intelectual, como, por ejemplo, Karl Marx, Miguel de Unamuno, Friedrich Nietzsche y Michael Foucault. Esto ha dado lugar al paso de la modernidad a la posmodernidad (Torres, 2002; Wesley, 2012), en la cual se relativizan los fundamentos racionalistas y empiristas. Así, se puede entender a la posmodernidad como un movimiento que «integra múltiples corrientes filosóficas que rechazan diferentes principios de la ilustración» (Mujica, 2020b, p. 796). El surgimiento de la posmodernidad también tuvo repercusiones en las teorías curriculares, puesto que contribuyen a superar el modelo curricular tecnológico-positivista, dando lugar a otros como el interpretativo cultural y el socio-crítico (Monzón, 2010). Además, sobre todo con el modelo interpretativo cultural y sus teorías constructivistas, surge la pedagogía o educación por competencias, la cual se ha extendido en los sistemas educativos de Europa y ha comenzado a tener presencia en América Latina (Gimeno-Sacristán, 2008; Valdés-Ayala, 2012). Y, por supuesto, también se ha integrado en el 
currículo escolar y universitario de EF, como es el caso de España (Blázquez, 2013; Lleixà, 2016; Pazo \& Tejada, 2015). En el caso de Chile, el enfoque por competencias se ha integrado en la educación superior (Pizarro, 2014), pero aún no en la educación escolar, por lo que se considera que aquello representa un retraso epistemológico en torno al currículum escolar chileno.

Con base en lo anteriormente expuesto, el presente ensayo tiene por objetivo analizar desde la perspectiva filosófica algunas problemáticas que existen en torno al currículum de EF escolar en Chile desde el tránsito de una perspectiva moderna a posmoderna y su lugar dentro del contexto social actual.

\section{Filosofía moderna y currículum chileno de Educación Física}

La filosofía racionalista, positivista y utilitarista sigue siendo la más representativa en el fondo de la fundamentación teórica del currículum escolar chileno. Esto se puede apreciar en diferentes aspectos, como que todavía se sigue teniendo una pedagogía por objetivos (Ministerio de Educación, 2016, 2018), que pone el foco pedagógico en unos contenidos didácticos definidos por la administración educativa del país de forma, principalmente, rígida y cerrada. Se señala que tienen una forma rígida porque el profesorado tiene obligatoriedad de abordarlos por medio de los objetivos verticales. También se señala que están establecidos de forma cerrada porque el profesorado no puede modificar los contenidos y objetivos propuestos por el Ministerio de Educación. No obstante, se reconoce que existe cierta flexibilidad para adaptar los objetivos al contexto educativo y al proyecto educativo institucional. Asimismo, que los objetivos están redactados de forma amplia, para que puedan incluir variadas actividades, por ejemplo, en el ámbito del deporte educativo. Cabe destacar, que aquella amplitud o ausencia de precisión, según Mujica \& Concha (2015), en algunos casos se traduce a ambigüedad en torno a los parámetros que son considerados adecuados para ser enseñados. En función de lo anterior, el currículo escolar chileno ha sido descrito de la siguiente forma:

El currículo escolar en Chile responde a un modelo pedagógico por objetivos, que esencialmente responde a un enfoque positivista de la educación, de modo que en cada asignatura disciplinar el profesorado tiene un listado de objetivos de aprendizaje (OA, en adelante) que desarrollar con su alumnado y el resultado de este proceso será evaluado en forma sumativa, asignando una nota a cada estudiante (Mujica, 2020c, p. 2).

El modelo curricular tecnocrático de la educación escolar chilena también se refleja en el «excesivo énfasis en los resultados académicos obtenidos en pruebas estandarizadas, tanto nacionales como extranjeras» (Moreno et al., 2014, p. 412). Aquel énfasis representaría la obsesión por la eficacia que tiene el enfoque de la pedagogía por objetivos (Gimeno-Sacristán, 2002). La asignatura de EF también se incluyó en la aplicación de pruebas estandarizadas a nivel nacional, por medio de un Sistema de Medición de la Calidad de la Educación (SIMCE) de EF. La orientación curricular de aquel SIMCE muestra uno de los grandes problemas curriculares, donde se aprecia una clara aplicación del dualismo cartesiano referido al cuerpo y al alma heredado de la filosofía moderna. En concreto, la aplicación es la pretensión de «evaluar la calidad de la EF a partir de la medición de los niveles de condición física y del índice de masa corporal en los estudiantes» (Moreno et al., 2014, p. 412). Reducir la calidad de la EF a lo que en epistemología de la EF se reconoce como capacidades físicas o condición física (Blázquez, 2006), es desconocer todos los aportes que se han hecho en torno al aprendizaje en EF (Cagigal, 1996; Castejón, 2010; Devís \& Peiró, 2007; Kirk, 2010; López-Ros et al., 2015; Mujica, 2021a, 2021c; Ruiz, 2021). En otras palabras, se diría que es el reflejo de una racionalidad curricular técnica en EF (Díaz, 2005). Por todo ello, Rodríguez (2016) ha señalado que medir la calidad de la EF en Chile es un desafío pendiente, lamentando que el actual SIMCE reproduzca una mirada equivocada de lo que es la calidad de dicha disciplina pedagógica. Buscando la causa de este errado enfoque tecnocrático en la EF chilena, se ha señalado lo siguiente:

Esta idea equivocada de la Educación Física, podría estar presente en el origen de la misma en el siglo XIX en Chile, sea a través de una fuerte influencia europea y sobre todo alemana, sea a través de la gimnasia de «Jahn», rígida y militarizada que pugna con la gimnasia sueca educativa promovida por Joaquín Cabezas por muchos años (Rodríguez, 2016, p. 178).

Frente a aquella propuesta reduccionista, se considera que, por un lado, debiese cambiarse el nombre medición de la calidad por el de evaluación de la calidad, puesto que el concepto correcto para abordar la calidad educativa es este último (Blázquez, 2006). En segundo lugar, es necesario desarrollar una nueva evaluación que considere la EF desde una mirada integral. Una propuesta que pretende aquello es la de Rodríguez et al. (2016), la cual propone las siguientes tres dimensiones: 
motriz, escolar y docente. Propuestas de este tipo, desde una mirada posmoderna o no tradicional, deberían ser discutidas y consensuadas con el profesorado de EF del sistema escolar para consolidar una evaluación más democrática. Sobre la propuesta de Rodríguez et al. (2016), se valora positivamente el enfoque integral que asume y la variedad de aspectos de la EF que son considerados, entre ellos la infraestructura y el currículum. Cabe destacar, que, actualmente, se encuentra suspendida la aplicación del SIMCE de EF, lo cual podría estar asociado a las críticas que se le han proporcionado o a los ajustes curriculares que se han generado por la pandemia. Es importante señalar también estas evaluaciones estandarizadas se encuentran fuertemente condicionadas por la desigualdad social y económica de la población chilena (Oliva, 2008). Los resultados de estas evaluaciones estandarizadas, si no son contextualizados y analizados desde una mirada crítica, pueden ser utilizados de forma perjudicial para los sectores más desfavorecidos.

De acuerdo a lo planteado anteriormente, es posible reconocer que la EF chilena todavía es fuertemente influenciada por la filosofía tradicional e higienista que predominaba en la enseñanza de la gimnasia en Europa durante el siglo XIX (Moreno, 2018: Mujica, 2021a), la cual tiene una fuerte carga de pedagogía conductista. Otro problema curricular de corte reduccionista en torno a la EF chilena es la de su actual nombre. Hace unos años la asignatura pasó de llamarse Educación Física a Educación Física y Salud. De este modo, se reflejó nuevamente la inclinación epistemológica hacia una concepción mecanicista e higienista que reduce la asignatura a los beneficios fisiológicos y psicológicos que puede producir. Es una mirada utilitarista de la asignatura, que se aleja de una comprensión compleja del aprendizaje. Se mantiene la univocidad de la lógica causa-efecto que predominó en la cultura científica positivista, distanciándose de una EF que promueva una mirada de las teorías con enfoque cultural y sociocrítico (Cagigal, 1996; Devís, 1996, 2006, 2012, 2018; Kirk, 2010, 2017; Mujica, 2020d). La lógica curricular de intensificar la relación de la EF con la salud la convierte en una disciplina de corte biomédico y psicologista, reduce la importancia en torno a la integralidad y las bases filosóficas que deben orientar a una adecuada pedagogía (Marina, 2005; Mujica \& Orellana, 2021; Prieto, 2018). Por dicha lógica se ha concluido que la EF chilena tiene las siguientes características epistémicas:

Una disciplina raciotécnica, dicotómica y reproductiva, basada esencialmente en fundamentos científicos como los fisiológicos, biomédicos o biomecánicos, que se contradice con las tendencias actuales de la disciplina que busca el desarrollo de una EF más crítica que reproductora de modelos ajenos al estudiantado (Moreno et al., 2014, pp. 423-424).

Con esta mirada reduccionista en torno a la salud se corre el riesgo de fomentar miradas obsesionadas con la salud, donde todo debe ser pensado en función de si ayuda o no ayuda al estado de salud, lo cual incluye una perspectiva filosófica utilitarista. De este modo lo importante de la EF sería ajeno a ella misma, pues la salud es un constructo epistémico independiente de dicha disciplina pedagógica. Asimismo, existe la probabilidad de que se estén generando estereotipos corporales muy fuertes en torno a la salud, los cuales pueden ser opresivos y discriminadores entre el estudiantado. Este fenómeno cultural en torno a la obsesión con la salud ha sido reconocido por Beltrán-Carrillo \& Devís-Devís (2019) como salutismo en EF, caracterizado por un discurso hegemónico que menosprecia cualquier corporalidad que se distancie de los estereotipos de salud promovidos de forma estandarizada. De acuerdo con dichos autores, el salutismo puede disminuir el interés del alumnado por la clase de EF, sobre todo, por las inadecuadas experiencias asociadas, desde la perspectiva ética. En términos más precisos han señalado que «el salutismo puede promover la insatisfacción corporal, la vergüenza, la marginación y los trastornos del comportamiento alimentario y de ejercicio de aquellos que no simbolizan el cuerpo sano y en forma» (Beltrán-Carrillo \& DevísDevís, 2019, p. 23).

Se puede apreciar una clara relación entre el salutismo y la filosofía moderna que separa cuerpo y alma, asumiendo una postura objetivista en torno a la corporalidad humana. Precisamente, sobre las bases epistemológicas de dicho fenómeno, se refiere a «una visión superficial que se queda en la forma corporal, como dimensión más material, objetiva y tangible del ser humano. De este modo, con el salutismo emergen asociaciones acríticas y simplistas entre la salud y la forma corporal» (Beltrán-Carrillo \& Devís-Devís, 2019, p. 23). En este sentido, la lógica curricular de la EF chilena ha sido establecida por miradas bastante acríticas en torno a lo que significa haber intensificado la relación de la EF con el ámbito de la salud. Cabe destacar, que la EF siempre estará ligada a dicho ámbito, de modo que haberle cambiado el nombre a la asignatura no era necesario para que exista dicho vínculo. No obstante, hacer ese cambio era necesario para discriminar otros importantes constructos de la EF, como la recreación, 
el deporte, la motricidad, la diversidad, la ética o la interculturalidad (Lleixà, 2002; Ríos, 2002).

\section{Filosofía posmoderna y currículum chileno de Educación Física}

Los principios filosóficos de la modernidad que dieron vida a la escuela moderna generaron una crisis educativa, sobre todo, frente a las variadas críticas y reflexiones filosóficas que promovían repensar las bases teóricas de la educación conductista (Pastor, 2005; Torrebadella, 2013a, 2013b, 2015, 2017, 2020). Asimismo, por los hallazgos y planteamientos provenientes de las ciencias particulares que contradecían aquel paradigma, como los de Piaget (1991), Vygotsky (1995), Bruner (1997) o Ausubel (2002). Así, en el último cuarto del siglo XX, ya había claridad de que en el mundo el modelo curricular por objetivos se derrumbaría y daría paso a otros modelos posmodernos (Gimeno-Sacristán, 2002, 2008, 2010). De este modo, surgía una teoría curricular posmoderna que «se caracteriza por la pluralidad, la diferencia, y sobre todo los discursos múltiples» (Torres, 2002, p. 200). Desde aquella teoría, como bien explica Monzón (2010), surgen modelos que promueven una enseñanza abier ta, flexible, contextualizada, política, crítica, reflexiva e histórica-cultural. En EF también sucede algo similar, pues surgen modelos alternativos a la enseñanza técnica y conductista, como el modelo de enseñanza comprensiva del deporte (López-Ros et al., 2015); el modelo de educación deportiva (Segovia \& Gutiérrez, 2019); el modelo de responsabilidad social del deporte (Hellison, 1995); y el modelo de enseñanza basada en el amor (Mujica, 2020a). También surgen teorías alternativas a la mirada positivista de la EF, como la psicomotricidad (Le Bouch, 1992), la ciencia de la motricidad humana (Sérgio, 2006), la praxiología motriz (Parlebas, 2001), la EF crítica (Kirk, 2010) y la EF poscartesiana (Mujica, 2021a). En términos generales, se aprecia que ha habido una influencia de estas perspectivas en el nuevo léxico de la disciplina, sobre todo, con la inclusión del término motricidad y sus diferentes manifestaciones. No obstante, se desconoce en términos más exactos la influencia de cada una de estas perspectivas. Lo cual se considera como una importante línea de investigación.

Las nuevas perspectivas posmodernas se han introducido más intencionadamente en la formación del profesorado de EF en Chile (Castillo-Retamal et al., 2020), incluyendo la teoría general de la educación y la teoría específica de la disciplina pedagógica (Kirk, 2010, 2017;
Monzón, 2010; Mujica, 2020a, 2020b). Se han concretado algunos avances a nivel de Educación Superior para configurar un currículum y una didáctica heredera de la posmodernidad que promueva metodologías de aprendizaje activo, de pensamiento crítico y reflexivo, así como de inclusión con los grupos humanos históricamente discriminados (Pizarro, 2014). Por ello, se espera que aquello contribuya en el desarrollo de la mirada de igualdad de género, de actividad física adaptada y de interculturalidad. Sin embargo, pueden surgir dificultades, dado que el currículum escolar de EF todavía no da grandes pasos para renovar su paradigma (Moreno, 2018; Mujica, 2020d; Rannau, 2020). Por ello, las posibilidades de innovar se verán limitadas a las posibilidades que otorgue dicho currículum enfocado por objetivos de aprendizaje. La mayor posibilidad que se tendrá de innovar es por medio de la didáctica, puesto que el currículum escolar de EF en Chile otorga más libertades sobre la forma de concretar el currículum. De este modo, el futuro profesorado de EF podrá introducir de algún modo las nuevas perspectivas y modelos en torno al currículum, a la espera de que exista un avance más significativo en torno al currículum. Aquel avance es fundamental, puesto que «mientras el currículo chileno y, por consiguiente, de EF, mantenga una lógica tecnocrática o economicista de la educación, es muy complejo emprender procesos pedagógicos que mantengan una lógica crítica y emancipadora» (Mujica, 2020d, p. 13).

Otro avance que exigirá una mirada posmoderna de la EF, es discutir más ampliamente el cambio de nombre de la asignatura, debido a que, según Cornejo \& Matus (2013), el excesivo enfoque por la salud le hace perder su profundo sentido pedagógico, lo cual ha sido expresado de este modo:

Desde la perspectiva del estado los nuevos programas curriculares de educación física de los escolares chilenos se basan en una Educación Física escolar orientada hacia la Salud, quedando demostrado que hoy día la educación física tiene una fuerte tendencia a medicalizar y perder el sentido educativo y de juego como ha sido concebido a través del tiempo (p. 23).

Abordar con rigurosidad estas problemáticas curriculares sería uno de los desafíos de una EF que trascienda la lógica tecnocrática, biomédica y mecanicista. Además, se considera que una educación por competencias, la cual es muy probable que se intente desarrollar en los próximos años, requerirá una mirada más integral de la EF. 


\section{Conclusiones y aplicaciones prácticas}

Con relación al objetivo de este ensayo, que fue analizar algunas problemáticas que existen en torno al currículum de EF escolar en Chile, se reconocen tres principales asuntos curriculares conflictivos. El primer problema surge de la evaluación de la calidad de la EF escolar en función de la condición física. A pesar de que actualmente no se encuentra en proceso de aplicación, es un sistema de evaluación reciente y refleja la perspectiva filosófica que ha predominado al momento de diseñarla.Y, en efecto, la interpretación que ha existido sobre lo que se entiende en el país por EF. Es posible que una discusión más amplia y que incluya una filosofía más crítica sobre la EF pueda ayudar a generar una evaluación más integral.

El segundo conflicto en torno al currículo escolar, sería la intencionalidad de asociar la EF a la salud por sobre otros ámbitos culturales, lo cual se refleja en el nuevo nombre de la asignatura. Este nuevo nombre genera el riesgo de que en la asignatura de EF se asuma una postura biomédica o higienista que reduzca la calidad de la EF al desarrollo de indicadores cuantitativos sobre la condición física. Este nuevo nombre sería un reflejo de la influencia positivista en la EF chilena. Y, finalmente, el tercer problema curricular surge por el retraso en el cambio de paradigma escolar, ya que en la formación inicial del profesorado de EF existe una educación por competencias y en las instituciones escolares una educación por objetivos. Sobre todo, porque una educación por competencias podría contribuir a renovar la filosofía de la educación de la administración estatal y de la administración de las escuelas. De este modo, se concluye que la EF escolar en Chile tiene grandes desafíos curriculares para poder incluir las innovaciones que han permitido las nuevas teorías y modelos de la posmodernidad. Principalmente, el pasar de una educación por objetivos a una educación por competencias.

Dado que este es un trabajo académico de corte teórico, se considera adecuado pensar aplicaciones que puedan desarrollarse también en dicho contexto intelectual. En este sentido, se considera que una aplicación de este ensayo puede ser la de generar grupos de discusión en las asignaturas de currículum de la formación de docentes en EF, con la finalidad de generar propuestas pedagógicas orientadas a progresar hacia una epistemología posmoderna. Asimismo, también podría ser aplicado en actividades pedagógicas similares en los programas de posgrado que aborden la filosofía de la EF. Otra aplicación es que el contenido de este artículo pueda ser debatido entre los especialistas de EF que se desempeñan en el Ministerio de Educación, con el propósito que se puedan abrir instancias que permitan la renovación curricular de la asignatura en la etapa escolar. Aquello podría contribuir a la necesidad que se genere un amplio debate en torno a la materia, el cual cuente con una amplia participación del profesorado y del alumnado. Esto, a su vez, favorecería la democratización del currículum.

\section{Futuras líneas de investigación}

Se reconocen las siguientes cinco líneas de investigación: a) indagar qué propuestas de evaluación de la calidad de la EF existe en las diferentes universidades chilenas; b) investigar la posible presencia de conductas salutistas en los contextos de EF escolar en Chile; c) analizar la percepción del profesorado de EF titulado que se ha formado en una educación por competencias sobre las posibilidades que le ofrece el currículum escolar por objetivos; d) identificar, desde diferentes perspectivas, la presencia de las nuevas corrientes epistemológicas de la EF en el currículo escolar chileno; y e) establecer el porcentaje de objetivos que tributan a la dimensión de condición física en el currículum escolar de EF en Chile.

\section{Referencias}

Arango, S. (2008). Goethe y el romanticismo alemán. Lingüistica y Literatura, 53, 51-64.

Ausubel, D. (2002). Adquisición y retención del conocimiento: una perspectiva cognitiva. Barcelona: Paidós.

Beuchot, M. (1993). Cuerpo y alma en el hilemorfismo de Santo Tomás. Revista Española de Filosofía Medieval, 0, 39-46. doi: 10.21071/refime.v0i.9931

Blázquez, D. (2006). Evaluar en Educación Física (9a ed.). Barcelona: Inde.

Blázquez, D. (2013). Diez competencias docentes para ser mejor profesor de Educación Física. Barcelona: Inde.

Bruner, J. (1997). La educación, puerta de la cultura. Madrid: Visor.

Bunker, D., \& Thorpe, R. (1982). A model for the teaching of games in the secondary school. Bulletin of Physical Education, 10, 9-16.

Cabedo, C. (2006). Filosofía y cultura de la tolerancia. Castelló de la Plana: Universitat Jaume.

Cagigal, J. M. (1996). Obras Selectas.Volumen III. Madrid: Comité Olímpico Español.

Casillas-Gutiérrez, C. (2019). Currículum, ideología y 
capacidad crítica en la docencia universitaria. Revista Educación, 43(1), 698-733. 2019. doi: 10.15517/ revedu.v43i1.30728

Castejón, F. J. (Ed.) (2010). Deporte como concepto y aplicación. En F. J. Castejón (Ed.), Deporte y Enseñanza Comprensiva (pp. 11-34). Sevilla: Wanceulen.

Castillo-Retamal, F., Almonacid-Fierro, A., CastilloRetamal, M., \& Bássoli de Oliveira, A. (2020). Formación de profesores de Educación Física en Chile: una mirada histórica (Physical Education teacher training in Chile: a historical view). Retos, 38, 317 324. https: / / doi.org/10.47197/retos.v38i38.73304

Contreras, O. (1998). Didáctica de la Educación Física: Un enfoque constructivista. Barcelona: Inde.

Cornejo, M., \& Matus, C. (2013). Educación Física en Chile. Revista Electrónica Actividad Física y Ciencias, 5(1), $1-25$.

Descartes, R. (2003). Meditaciones metafísicas. Madrid: Gredos.

Devís, J. (1996). Educación Física, deporte y currículum. Investigación y desarrollo curricular. Madrid: Visor.

Devís, J. (2006). Bases para una propuesta de cambio en la enseñanza de los juegos deportivos. En L. Brito., L. Palacios., C. Martínez y R. Tinoco (Eds.), Educación Física I (pp. 65-76). México: Secretaría de Educación Pública.

Devís-Devís, J. (2012). La investigación sociocrítica en la educación física. Estudios Pedagógicos, 38 (especial), 125-153. doi: 10.4067/S0718-07052012000400008

Devís, J. (2018). Los discursos sobre las funciones de la Educación Física Escolar. Continuidades, discontinuidades y retos. Revista Española de Educación Física y Deportes, 423, 121-131.

Devís, J., \& Peiró, C. (2007). La iniciación en los juegos deportivos: la enseñanza para la comprensión. En R. Arboleda (Ed.), Aprendizaje motor: Elementos para una teoría de la enseñanza de las habilidades motrices (pp. 105 129). Medellín: Funámbulos.

Dewey, J. (2004). Democracia y educación. Una introducción a la filosofía de la educación.

Díaz, F. (2005). La evaluación formativa como instrumento de aprendizaje en Educación Física. Barcelona: Inde.

Esparza, G.A., \& Zavala, G. M. (2015). ¿Marco curricular de enseñanza o aprendizaje significativo? Una visión conciliadora dentro del aula. DOCERE, (13), 7-10. doi: 10.33064/2015docere131749

Ferrater, J. (1994). Diccionario de filosofía de bolsillo (AH). Madrid: Alianza.

Gallo, L. (2009). El cuerpo en la educación da qué pensar: perspectivas hacia una educación corporal. Revista
Estudios Pedagógicos, 35(2), 232-242. doi: 10.4067/ S0718-07052009000200013

García, M. (1999). Introducción filosófica a la educación física. Tendencias Pedagógicas, 4, 129-140. Recuperado de https://revistas.uam.es/ tendenciaspedagogicas/article/view/1798

García, M. (2014). Filosofía y actitud filosófica: sus aportaciones a la educación. Revista Española de Pedagogía, 72(258), 231-247.

Gimeno-Sacristán, J. (2002). La pedagogía por objetivos: obsession por la eficiencia (1 $1^{\mathrm{a}} \mathrm{ed}$.). Madrid: Morata.

Gimeno-Sacristán, J. (2008) (Ed.). Educar por competencias, ¿qué hay de nuevo? Madrid: Morata.

Gimeno-Sacristán, J. (2010). ¿Qué significa el currículum? E. J. Gimeno-Sacristán (ed.), Saberes e incertidumbres sobre el currículum (21-44). Madrid: Morata.

González, A., \& Hernández, A. (2014). Positivismo, Dialéctica Materialista y Fenomenología:Tres Enfoques Filosóficos del Método Científico y la Investigación Educativa. Actualidades Investigativas en Educación, 14(3), 502-523.

Hellison, D. (1995). Teaching responsibility through physical activity. Champaign, IL: Human Kinetics.

Iskandar, J., \& Rute, M. (2002). Sobre positivismo e educação. Revista Diálogo Educacional, 3(7), 89-94.

Kant, I. (1989). La metafísica de las costumbres. Madrid: Tecnos.

Kirk, D. (2010). Physical Education Futures. New York: Routledge.

Kirk, D. (2017). Teaching Games in Physical Education. Towards a pedagogical model. Revista Portuguesa de Ciências do Desporto, Supl. 1, 17-26. doi: 10.5628/ rpcd.17.s1a. 17

Lawson, H. (2009). Paradigms, exemplars and social change. Sport, Education and Society, 14(1), 97-119. doi: 10.1080/13573320802615247

Lawson, H. (2018). Physical Education in the industrial age school. En H. Lawson (Ed.), Redesigning Physical Education: An equity agenda in which every child matters (pp. 23-40). New York: Routledge.

Lawson, H. (2019). The Physical Education System as a Consequential Social Determinant. Quest, 72(1), 72-84. doi:10.1080/00336297.2019.1627224

Le Boulch, J. (1992). Hacia una ciencia del movimiento humano. Introducción a la psicokinética. Barcelona: Paidós.

Locke, J. (1984). Ensayo sobre el entendimiento humano. Madrid: Sarpe.

López-Ros, V. (2016). El «modelo integrado técnicotáctico» de enseñanza deportiva. Origen, contextualización y características metodológicas. 
Ejournal de la Recherche Sur l'intervention en Éducation Physique et Sport, 38, 63-91. doi: 10.4000/ejrieps.892

López-Ros, V., Castejón-Oliva, F. J., Bouthier, D., \&Llobet-Martí, B. (2015). Modelos para una enseñanza comprensiva del deporte. Espacios comunes para el encuentro (y algún desencuentro). Revista Ágora para la Educación Física y el Deporte, 17(1), 45 60.

Lleixà, T. (2016). ¿Qué es realmente esto de las competencias? EnT. Lleixà y E. Sebastiani (Eds.), Competencias clave y Educación Física (pp. 19-30). Barcelona: Inde.

Matus, C. (2016). Los usos del afecto en el currículo escolar. Educação em Revista, 32(2), 111-130. doi: 10.1590/0102-4698153449

Marina, J. (2005). Precisiones sobre la educación emocional. Revista Interuniversitaria del Profesorado, 54, $27-$ 44.

Ministerio de Educación. (2016). Educación Física y Salud. Programa de Estudio de Primero Medio. Santiago: República de Chile.

Ministerio de Educación. (2018). Bases Curriculares. Primero a sexto básico. Santiago: República de Chile.

Molina, M., Gutiérrez, D., Segovia, Y., \& Hopper, T. (2020). El modelo de Educación Deportiva en la escuela rural: amistad, responsabilidad y necesidades psicológicas básicas. Retos, 38, 291-299. https: / / doi.org/10.47197/retos.v38i38.73685

Monzón, L. (2010). El currículo como mediación cultural: una perspectiva hermenéutico-analógica. Revista Latinoamericana de Estudios Educativos, 40(2), 37-58.

Moreno, A. (2018). La Educación Física chilena en educación básica: Una caracterización crítica. The Journal of the Latin American Socio-cultural Studies of Sport (ALESDE), 9(2), 65-78.

Moreno, A., Gamboa, R., \& Poblete, C. (2014). La Educación Física en Chile: análisis crítico de la documentación ministerial. Revista Brasileira de Ciências do Esporte, 36(2), 411-427. https: / / doi.org/10.1590/ S0101-32892014000200010.

Moreno, A., \& Poblete, C. (2015). La educación física chilena y su profesorado: proponiendo algunos retos para la investigación en el área (Chilean physical education and its teachers: proposal of some challenges for research about the subject). Retos. Nuevas Tendencias en Educación Física, Deportes y Recreación, 28, 291-296. https://doi.org/10.47197/ retos.v0i28.35651

Mujica, F. (2020a). Educación Física y Ética. Sevilla: Wanceulen
Mujica, F. (2020b). El término Educación Física en la posmodernidad: contribución de algunas perspectivas fenomenológicas. Retos. Nuevas Tendencias en Educación Física, Deportes y Recreación, 38, 795-801. doi: 10.47197/retos.v38i38.73011

Mujica, F. (2020c). Análisis crítico del currículo escolar en Chile en función de la justicia social. Revista Electrónica Educare, 24(1), 1-14. doi: 10.15359/ree.241.25

Mujica, F. (2020d). Educación Física crítica: un enfoque fundamental para la igualdad de género y la inclusión educativa en Chile. Revista Infancia, Educación y Aprendizaje, 6(2), 1-17. doi: 10.22370/ ieya.2020.6.2.1374

Mujica, F. (2021a). Filosofía y Educación Física. Vigo: Mcsports.

Mujica, F. (2021b). Sobre la discusión filosófica del término educación física: una perspectiva ecléctica. Revista Dilemas Contemporáneos: Educación, Política y Valores, 9, 1-13. doi: 10.46377/dilemas.v9i1.2876

Mujica, F. (2021c). Emociones negativas del alumnado de Secundaria en el aprendizaje de baloncesto en Educación Física. Retos, 41, 362-372. doi: 10.47197/ retos.v0i41.84395

Mujica, F., \& Concha, R. (2015). Objetivos de aprendizaje de primer a sexto año básico en Educación Física y Salud: Análisis crítico de la documentación ministerial. Revista Motricidad Humana, 16(1), 18-25.

Mujica, F., \& Inostroza, C. (2020). Políticas educativas, evaluaciones estandarizadas y formación escolar integral en Chile: análisis de la desvalorización a diferentes materias del currículo. Dilemas Contemporáneos: Educación, Política y Valores, 7(2), 1-20. Scielo.

Mujica, F., \& Orellana, N. (2021). El amor en educación física: una perspectiva filosófica basada en Max Scheler y José Ortega y Gasset (Love in physical education: a philosophical perspective based on Max Scheler and José Ortega y Gasset). Retos. Nuevas Tendencias en Educación Física, Deportes y Recreación, 39, 857-862. doi: 10.47197/retos.v0i39.78787

Oliva, M.A. (2008). Política educativa y profundizacion de la desigualdad en chile. Estudios pedagógicos (Valdivia), 34(2), 207-226. doi: 10.4067/S071807052008000200013

Parlebas, P. (2001). Juegos, deportes y sociedades. Léxico de praxiología motriz. Barcelona: Paidotribo.

Pastor, J. L. (2005). Educación Física y libros de texto en la enseñanza primaria (1883-1978). Madrid: Dykinson.

Pazo Haro, C. I., \& Tejada Mora, J. (2015). Las competencias profesionales en Educación Física. Retos, 22, 
5-8. https://doi.org/10.47197/retos.v0i22.34575

Pérez, J. (2015). El positivismo y la investigación científica. Revista Empresarial, 9(3), 29-34.

Piaget, J. (1991). Seis estudios de psicología. Barcelona: Labor.

Pizarro, I. (2014). El modelo de educación por competencias y su impacto en la planificación estratégica de la Universidad de Talca (Chile). Revista Universitaria Ruta, 16(1), 7-18. Recuperado a partir de https://revistas.userena.cl/index.php/ruta/ article/view/466

Platón. (1988). Diálogos III. Fedón. Banquete. Fedro (1 reimpresión). Madrid: Gredos.

Prieto, M. (2018). La psicologización de la educación: implicaciones pedagógicas de la inteligencia emocional y la psicología positiva. Educación XX1, 21(1), 303-320. doi: 10.5944/educXX1.16058

Rannau, J. P. (2020). Educación Física en Chile: hacia una transdiciplinariedad desde el currículum y la colaboración pedagógica. Praxis Educativa, 24(2), 1-17.

Ríos, M. (2002). Manual de Educación Física Adaptada al alumnado con discapacidad. Barcelona: Paidotribo.

Rodríguez, F. (2016). Medición de la calidad de Educación Física en Chile, un desafío pendiente. Espacios en Blanco. Revista de Educación, 26, 173-185.

Rodríguez, F., Curilem Gatica, C., Escobar Gómez, D., \& Valenzuela Eberhard, L. (2016). Propuesta de evaluación de la educación física escolar en Chile. Educación Física y Ciencia, 18(1), 1-12.

Rojas, C. (2006). La filosofía. Sus transformaciones en el tiempo. San Juan: Isla Negra.

Rousseau, J. J. (2011). Emilio o de la educación. Madrid: Alianza.

Ruiz, L. M. (2021). Educación Física y baja competencia motriz. Madrid: Morata.

Sánchez, D. (2010). El positivismo, el empirismo lógico y las ramas de la matemática que incidieron en la geografía. Revista digital del Grupo de Estudios sobre Geografía y Análisis Espacial con Sistemas de Información Geográfica, 2(2), 20-53.

Segovia, Y., \& Gutiérrez, D. (2019). Esfuerzo, Implicación y Condición Física percibida en un Programa HIIT en Educación Física. Modelo Educación Deportiva vs Metodología Tradicional (Perception of Exertion, involvement and physical fitness in a HIIT Program in Physical Education. Sport Edu. Retos, 38, 151-158. https://doi.org/10.47197/ retos.v38i38.73686

Sérgio, M. (2006). Motricidad Humana, ¿Cuál es el futuro? Pensamiento Educativo, 38, 14-33.
Siedentop, D. (1994). Sport Education: Quality PE through positive sport experiences. Champaign, IL: Human Kinetics.

Stein, E. (2005). Obras completas II. Escritos filosóficos (Etapa fenomenológica: 1915-1920). Burgos: Monte Carmelo.

Stolz, S. (2013). Phenomenology and Physical Education. Educational Philosophy andTheory, 45(9), 949-962. doi: 10.1080/00131857.2013.785355

Stolz, S. (2014). The Philosophy of Physical Education. A new Perspective. London: Routledge

Torrebadella, X. (2013a). Gimnástica y educación física en la sociedad española de la primera mitad del siglo XIX. Lleida: Universitat de Lleida.

Torrebadella, X. (2013b). Cuerpos abandonados y rescatados: la educación física en los orfanatos españoles del siglo XIX. Cabás, 10, 11-28.

Torrebadella, X. (2015). Los batallones infantiles en la educación física española (1890-1931). ODEP. Revista Observatorio del Deporte, 1(1), 32-70.

Torrebadella, X. (2017). La militarización de la educación física escolar: análisis de dos imágenes publicadas en la prensa de Barcelona de principios del siglo XX. Historia Social y de la Educación, 6(1), 78-108. doi:10.17583/hse.2017.2393

Torrebadella, X. (2020). La escolarización de la educación física: un análisis de cinco imágenes publicadas en la prensa de Barcelona de principios del siglo XX (1910-1913). Revista Brasileira de Historia da Educação, 20, 1-30.

Torres, J.A. (2002). ¿Existe un currículum postmoderno? De las teorías curriculares de la modernidad al currículum postmoderno. Revista Innovación Educativa, 12, 197-208.

Tredici, J. (1958). Historia de la filosofía (4 $4^{a}$ ed.). Buenos Aires: Difusión.

Valdés-Ayala, Z. (2012). Constructivismo en educación. Ilusiones y dilemas. Calidad en la Educación Superior, $3(1), 24-42$.

Vilanou, C. (2015). Pensamiento Pedagógico en el idealismo alemán y en Schleiermacher. Historia y Memoria de la Educación, 1, 497-502. doi: 10.5944/ hme.1.2015.13206

Von Hildebrand, D. (2000). ¿Qué es filosofía? Madrid: Encuentro.

Vygotsky, L. (1995). Pensamiento y lenguaje. Teoría del desarrollo cultural de las funciones psíquicas. Bogotá: Fausto

Wesley, T. (2012). Posmodernidad y educación cristiana: desafíos ideológicos contemporáneos. Enfoques, 24(2), 85-100. 